\title{
HDL inhibited atherosclerosis induced by radiation injury
}

\author{
Jin Xie ${ }^{\mathrm{a}, \mathrm{b}, \dagger}$, Ke Zhu ${ }^{\mathrm{c}, \dagger}$, Qingya Wang ${ }^{\mathrm{a}}$, Pei Zhao ${ }^{\mathrm{a}}$, Lihua Pan ${ }^{\mathrm{a}}$, Jie Hui ${ }^{\mathrm{a}, *}$ \\ a The First Affiliated Hospital of Soochow University, Suzhou 215006 China \\ b Jingzhou Central Hospital, Jingzhou 434023 China \\ c Xuzhou Central Hospital, Xuzhou 221000 China \\ *Corresponding author, e-mail: 519274227@qq.com \\ $\dagger$ These authors contributed equally to this work.
}

Received 17 Jan 2021

Accepted 30 Aug 2021

\begin{abstract}
High-density lipoprotein (HDL) inhibits atherosclerosis development from radiation damage; nonetheless, the underlying mechanism is yet to be defined. This work studied patients treated with radiation along with cultured mouse aortic endothelial cells (MAECs) to investigate the process. Firstly, 158 patients who received radiation after neck cancers participated, and their arterial function was monitored by a B ultrasound. Similarly, HDL and other blood lipid indexes were also investigated. Then, MAECs were isolated, cultured, and passed through MTT assay to test the HDL protective role on ultraviolet B (UVB) radiation along with western blotting to test some apoptosis protein expression and possible molecules. Patients with high HDL levels were significantly less likely to develop atherosclerosis. We observed that MAECs treated with UVB were damaged significantly. However, HDL reversed the cell damage in a dose-depended manner. Meanwhile, the apoptosis process was investigated, and it revealed that HDL prevented UVB-induced apoptosis. Western blotting results showed that HDL enhanced phosphatidylinositol 3-kinase (PI3K) in addition to AKT phosphorylation in MAECs. These findings imply that HDL protects against UVB-induced apoptosis via activating the PI3K/AKT signaling pathway.
\end{abstract}

KEYWORDS: atherosclerosis, radiation, endothelial cells, high-density lipoprotein

\section{INTRODUCTION}

Atherosclerosis affects large arteries and has been one of the major causes of heart diseases [1]. It can cause hardening of the artery wall, narrowing of the lumen, and loss of elasticity of the middle membrane, resulting in major complications such as ischemic heart disease (IHD), myocardial infarction (MI), stroke (cerebral thrombosis and cerebral hemorrhage), and limb gangrene. Many factors are considered responsible for both occurrence and development of atherosclerosis. However, hyperlipidemia and obesity caused by high-fat diet leading to cholesterol accumulation are known to be the major risk factors of atherosclerosis $[2,3]$. The incidence of atherosclerosis occurs earlier in hypertensive patients as compared to patients of the same age and sex who do not have hypertension. Furthermore, smoking remains one of the risk factors of atherosclerosis and has been the principal independent risk factor for coronary heart diseases. Research data reveal that heavy smoking can damage vascular endothelial cells in addition to a surge in the level of carboxyhemoglobin [4]. The two important complications of atherosclerosis are diabetes and hyperinsulinemia, and they are related to secondary hyperlipidemia. When the level of insulin in the blood is elevated, the content of HDL is lowered which leads to higher morbidity and mortality from coronary heart diseases [5]. Another strong independent risk factor for atherosclerosis is family history with genetic factors. The patients with familial hypercholesterolemia and familial lipoprotein lipase deficiency had a relatively higher incidence of atherosclerosis when compared with the control group $[6,7]$. According to the age-related pathological data, atherosclerosis is a gradually developing process from infancy. The rate of detection as well as the severity of atherosclerosis are augmented with age owing to age-related changes in the arterial wall. Before menopause, the rate of incidence of coronary atherosclerosis remained lower in women than men of the same age because women had a higher level of HDL and lower level of lowdensity lipoprotein (LDL). This difference between the two sexes vanished after menopause which may be ascribed to the effect of estrogen [8]. When the body is exposed to radiations, whether naturally or medically, they can induce damage and atherosclerosis. Similarly, radiations at high-altitude areas are responsible for causing atherosclerosis [9]. Exposure to such radiation aggravates the risk of coronary artery disease (CAD) along with atherosclerosis and is dependent upon the dose of radiation [10]. Therefore, the risk of atherosclerosis caused by coronary angiography as well as the radiation doses received by patients need to be evaluated [11]. Moreover, patients with esophageal cancer who were treated with radiations had a higher risk of heart complications which largely encompass pericardial disease along with coronary artery atherosclerosis in addition to valvular heart disease, cardiomyopathy, and arrhythmia [12]. As 
a result, there is a pressing need to learn how to avoid the risk of atherosclerosis during imaging or radiotherapy.

HDL has been shown to prevent or relieve atherosclerosis through a variety of mechanisms $[13,14]$. However, the role and mechanisms of HDL in mitigating radiation-induced atherosclerosis have yet to be discovered. This study aims to look into the association between the ratio of atherosclerosis in patients treated with radiation and plasma lipid levels, and then to look into the involvement of HDL and probable mechanisms using MAECs that have been exposed to radiation.

\section{MATERIALS AND METHODS}

\section{Patients}

In total, 158 patients from the Oncology Department of Jingzhou Central Hospital (Jingzhou, Hubei, China) who received radiation after neck cancers participated, and their arterial function was monitored by a B ultrasound. Similarly, HDL and other blood lipid indexes were also tested. All patients provided written informed consent.

\section{Animals}

C57BL/6 J mice were used for MAEC isolation. DMEM medium was obtained from Gibco Company, New York, USA. The Institutional Animal Care and Use Committee of the Soochow University approved the experiments that involved the use of animals. The Ethics Committee of Soochow University approved the present study protocol (clearance No. 2019854268). Furthermore, the Institutional guidelines for the care and use of laboratory animals were complied with while performing all animal experiments in addition to the employment of laboratory animals and that animals were anesthetized and killed using acceptable techniques.

\section{Cell culture}

C57BL/6 J mice were employed for isolating MAECs by applying an outgrowth technique which has been described earlier $[15,16]$. DMEM medium containing $10 \%$ calf serum was employed for maintaining MAECs at $37^{\circ} \mathrm{C}$ in a humidified incubator with $5 \% \mathrm{CO}_{2}$. For all of the experiments, serum-free DMEM medium was utilized to perform overnight (12-16 h) serum starvation of MAEC cells after they had grown to nearconfluence.

\section{Preparation of HDL}

Following the procedure, HDL was prepared [17]. Briefly, Human serum obtained from Jingzhou Central Hospital was taken to overlay with potassium bromide $(\mathrm{KBr})$ gradient solution that possessed a density of $1.063 \mathrm{~g} / \mathrm{ml}$. After that, these samples were ultracentrifuged at $35000 \mathrm{rpm}$ for $18 \mathrm{~h}$ to remove low and very low-density lipoproteins. The infranatant was adjusted to $1.21 \mathrm{~g} / \mathrm{ml}$ with solid $\mathrm{KBr}$ and blended with the $1.21 \mathrm{~g} / \mathrm{ml}$ buffered $\mathrm{KBr}$ solution, followed by centrifugation for $48 \mathrm{~h}$ at $48000 \mathrm{rpm}$. After collecting HDL, it was dialyzed 3 times against phosphate buffer saline (PBS) containing $1 \mathrm{mM}$ EDTA for $48 \mathrm{~h}$ at $4{ }^{\circ} \mathrm{C}$. Finally, it was passed through PBS for dialysis with no EDTA for $8 \mathrm{~h}$ and then filtered with the help of a 0.22 mm filter.

\section{MTT assay}

MTT assay was performed for measuring cell growth. Cells were seeded at a density of $5 \times 10^{3}$ cells per well in a 96-well plate and cultured for predetermined periods. At each time interval, the medium was replaced with a fresh cell culture medium containing $0.5 \mathrm{mg} / \mathrm{ml}$ MTT for $4 \mathrm{~h}$. Then, $150 \mu \mathrm{l}$ DMSO was added to each well and shaken on the low-speed rotation for $10 \mathrm{~min}$ until the crystal was fully dissolved, then measurement at $490 \mathrm{~nm}$ was performed by employing a Multiskan MS ELSA reader (xMark Microplate Absorbance Spectrophotometer, BioRad, CA, USA). For normalizing the relative cell number, the absorbance from control cells was used.

\section{SDS-PAGE and Western blotting}

Western blot assay was performed over total protein by adopting the standard western blotting protocol (Molecular Clone, Edition II). Primary antibodies and the concentrations used were: from Santa Cruz Company, Santa Cruz, USA:PI3K (1:1500, sc374534), p-ERK (1:500, sc-7383), ERK (1:500, sc94), p-AKT (1:500, sc-7985R), AKT (1:500, sc-8312), and GAPDH (1:1000, sc-575); from CST Company, Boston, USA:cleaved caspase 3 (1:500, CST 9579); and from Abcam Company, London, England:ATF-4 (1:500, ab-25331). Furthermore, secondary antibodies already labeled with respective horseradish peroxidase (HRP) were applied, followed by performing enhanced chemiluminescence (ECL) detection as suggested by the manufacturer (Pierce company, Rockford, USA). For the analysis of the integrated density mean grey value of the band, the ImageJ software was employed, and calculation was performed for the corresponding relative expression ratio.

\section{Statistical analysis}

Data were expressed as means \pm SE. The two-tailed Student's $t$-test (for 2 groups) was used to assess the differences of means among multiple groups in addition to the analysis of variance (ANOVA, for $>2$ groups). $p \leqslant 0.05$ was considered statistically significant for all analyses.

\section{RESULTS}

\section{HDL level related to the ratio of atherosclerosis}

We found that 118 patients (74.7\%) of the total 158 patients treated with radiation had atherosclerosis with 
Table 1 Relationship between incidence of atherosclerosis and blood lipid.

\begin{tabular}{lccrr}
\hline Parameter & AS & Non-AS & $\chi^{2}$ & $p$-value \\
\hline Sex (female/male) & $39 / 79$ & $15 / 25$ & 0.102 & 0.749 \\
Age ( $\leqslant 50 />50)$ & $30 / 88$ & $26 / 14$ & 18.756 & $<0.001$ \\
Total cholesterol (mmol/L) & $7.38 \pm 0.82$ & $4.25 \pm 0.74$ & 21.364 & $<0.001$ \\
Triglyceride (mmol/l) & $3.85 \pm 0.68$ & $2.46 \pm 0.65$ & 11.295 & $<0.001$ \\
LDL (mmol/l) & $3.57 \pm 0.54$ & $2.83 \pm 0.48$ & 7.695 & $<0.001$ \\
HDL (mmol/l) & $1.35 \pm 0.43$ & $1.97 \pm 0.57$ & 7.226 & $<0.001$ \\
\hline
\end{tabular}

$\chi^{2}$ means value of chi square test.

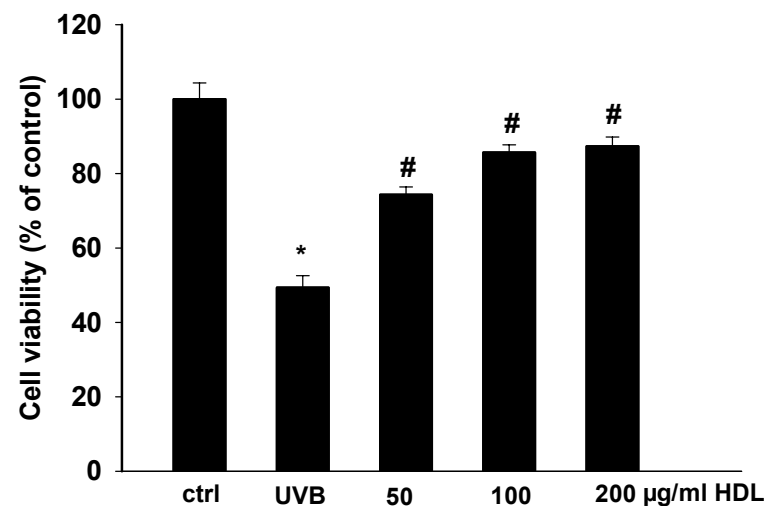

Fig. 1 Effects of HDL on MAEC cell viability. Results were confirmed in 3 independent experiments. * compared with control group, $p<0.05$; \# compared with UVB group, $p<$ 0.05 .

different degrees. Moreover, the incidence rate of atherosclerosis did not have a significant association with sex, but rather with age. Similarly, the results of the plasma lipid test revealed that patients with no atherosclerosis had a higher level of HDL than those with atherosclerosis, thus signifying that a high level of HDL can significantly reduce the probability of atherosclerosis development (Table 1).

\section{HDL reversed radiation-cell damage}

The MAECs were first irradiated with $90 \mu \mathrm{w} / \mathrm{cm}^{2} \mathrm{UV}$ rays for $10 \mathrm{~min}$ after which they were either treated with 50, 100, $200 \mu \mathrm{g} / \mathrm{ml} \mathrm{HDL}$, or left untreated. MTT results demonstrated that UVB caused about 52\% MAEC damage. However, HDL reversed the phenomenon, therefore this indicated that HDL protected MAEC damage caused by UVB (Fig. 1).

\section{HDL protected UVB-induced apoptosis}

In comparison to the control group or HDL group, the cleaved caspase 3 expression in the UVB group was increased. However, in the UVB group treated with $200 \mu \mathrm{g} / \mathrm{ml} \mathrm{HDL}$, the cleaved caspase 3 expression was lowered; this indicated that HDL alleviated UVBinduced apoptosis. ATF-4 as an apoptosis protein was related to apoptosis induced by endoplasmic reticulum stress. Thus, ATF-4 expression was elevated by
(A)

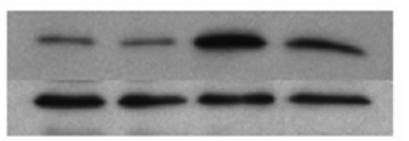

Cleaved

caspase-3

GAPDH

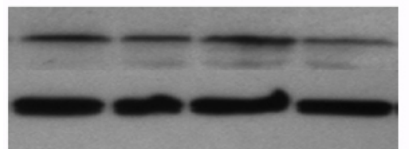

ATF-4

GAPDH

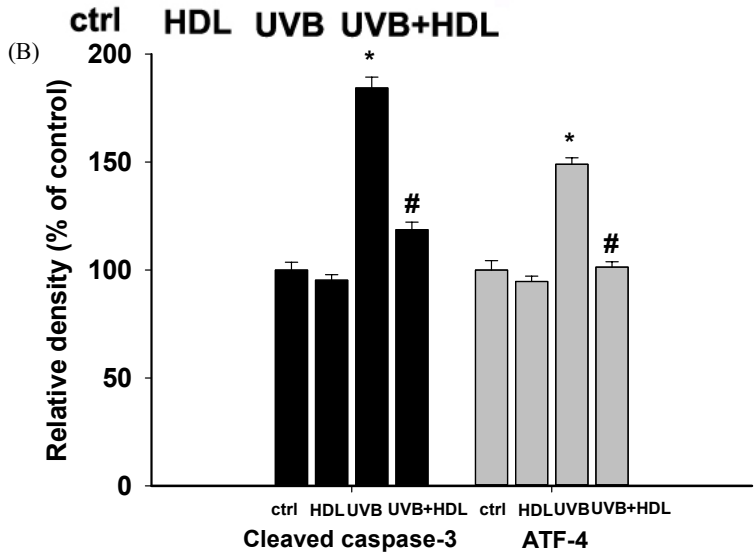

Fig. 2 HDL inhibited MAEC apoptosis caused by UVB. * compared with control group, $p<0.05$; \# compared with UVB group, $p<0.05$.

UVB while it was reversed by HDL during this study, indicating that HDL may protect MAECs by inhibiting endoplasmic reticulum stress apoptosis (Fig. 2).

\section{HDL inhibited radiation-induced atherosclerosis by PI3K/AKT signaling pathway}

To further explore the possible mechanism, this experiment was designed to study the influence of HDL over PI3K as well as AKT expression besides phosphorylation. Fig. 3 shows that the total amount of PI3K and AKT proteins was lowered by UVB while the total amount of these proteins was not altered by HDL treatment, but rather it enhanced their phosphorylation. However, PI3K and AKT were inhibited by LY294002. It can be inferred from these results that HDL promotes cholesterol transport in MAECs via a mechanism in 


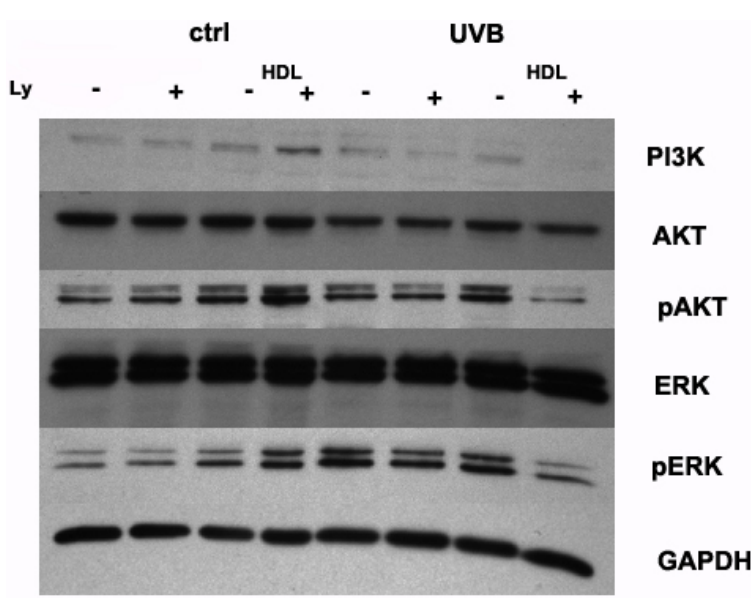

Fig. 3 HDL inhibited MAEC apoptosis mainly through activation of PI3K/AKT pathway.

which the PI3K/AKT pathway is triggered (Fig. 3).

\section{DISCUSSION}

Atherosclerosis causes many clinical problems such as angina, myocardial infarction, stroke, hemiplegia, aphasia, renal artery stenosis, and lower extremity artery stenosis. These are quite serious complications and can be fatal. Several factors contribute to the development of atherosclerosis, and radiation is one of them. Patients who have had tumors treated with radiation have also been diagnosed with atherosclerosis $[18,19]$. HDL has been shown to protect against atherosclerosis; however, whether it can protect against radiation-induced atherosclerosis is not fully known. Therefore, our study revealed that radiation can inflict heavy damage upon cells; however, such damage can be reversed when these cells are treated with HDL. Nonetheless, some studies have suggested that moderate ultraviolet radiation has the potential to prevent cardiovascular disease [20]. Notwithstanding ionizing radiation even in low doses will aggravate the risk of cardiovascular disease, primarily causing endothelial cell damage which leads to the process of atherosclerosis $[21,22]$. HDL reduces the deposition of cholesterol into tissues, thereby playing an antiatherosclerotic role by preventing the occurrence and development of atherosclerosis [23]. Our clinical studies demonstrated that radiation exposure remained a high-risk factor for the occurrence of atherosclerosis; however, HDL could effectively reduce such risk. Radiation can cause apoptosis by oxidative stress as evidenced by the increased apoptosis of MAECs during the cell experiment with irradiation, and HDL may prevent apoptosis [24]. However, our study exhibited that HDL inhibited the apoptosis of the endothelial cell [25-27]. Although some studies have found that UVB radiation resulted in endothelial cell apoptosis primarily by inhibiting PI3K/AKT signaling pathway $[28,29]$, and HDL can protect endothelial cells through PI3K/AKT signaling pathway [30], but it remained unclear whether HDL can protect endothelial cells from apoptosis caused by UVB radiation. In this study, HDL augmented PI3K/AKT expression in the UVB-treated group but could not eliminate the effect of PI3K/AKT inhibitor, LY294002. Therefore, this was consistent with the result that PI3K/AKT pathway is not the only driver in HDL-mediated cell protection [31]. Therefore, with further experimental confirmation, there is a high chance to translate the current result for clinical use to prevent radiotherapy-induced atherosclerosis.

\section{Study strength and limitations}

This study focused on HDL protective effects against radiation-induced atherosclerosis. It also provides some evidence for how to avoid or mitigate the adverse effects of radiation therapy for some cancer patients as well as some guidance for radiology staff members on how to reduce the incidence of AS. However, more animal model tests are needed to elucidate the significance of HDL as well as collaboration with nutritionists to provide radiation safety measures.

\section{CONCLUSION}

Radiation-induced AS is gaining more and more attention from workers exposed to radiation and radiation therapy patients. HDL as a potential drug plays a significant role in preventing or attenuating radiation-induced AS. This work first established that HDL protects against UVB-induced AS via activating the PI3K/AKT signaling pathway.

Acknowledgements: This study was supported by the natural science foundation of Hubei province (2017CFB786), the medical school Youth Fund of Yangtze University (YXYQ201411), and the Jingzhou Science and Technology Bureau Project (2017-93). The authors would like to thank all the reviewers who participated in the review and MJEditor (www.mjeditor.com) for its linguistic assistance during the preparation of this manuscript.

\section{REFERENCES}

1. Meng XD, Yao HH, Wang LM, Yu M, Shi S, Yuan ZX, Liu J (2019) Knockdown of GAS5 inhibits atherosclerosis progression via reducing EZH2-mediated ABCA1 transcription in ApoE-/- mice. Mol Ther Nucleic Acids 19, 84-96.

2. Miao J, Zang X, Cui X, Zhang J (2020) Autophagy, hyperlipidemia, and atherosclerosis. Adv Exp Med Biol 1207, 237-264.

3. Zhu L, Guo G, Fan Z-Q, Wang N, Zou D-Q, Shi X-Q (2021) Alleviation of high-fat-diet induced obesity and cholesterol accumulation in mice by extracts from male zooid of Antheraea pernyi. ScienceAsia 47, 162-169.

4. Boua PR, Brandenburg JT, Choudhury A, Hazelhurst S, Sengupta D, Agongo G, Nonterah EA, Oduro AR, et al (2020) Novel and known gene-smoking interactions 
with cIMT identified as potential drivers for atherosclerosis risk in West-African populations of the AWI-Gen study. Front Genet 10, ID 1354.

5. Chen HC, Lee WC, Fang HY, Fang CY, Chen CJ, Yang $\mathrm{CH}$, Wu CJ (2020) Impact of high triglyceride/highdensity lipoprotein cholesterol ratio (insulin resistance) in ST-segment elevation myocardial infarction. Medicine (Baltimore) 99, e22848.

6. Shiomi M (2020) The history of the WHHL rabbit, an animal model of familial hypercholesterolemia (II) contribution to the development and validation of the therapeutics for hypercholesterolemia and atherosclerosis. J Atheroscler Thromb 27, 119-131.

7. Zhao H, Li Y, He L, Pu W, Yu W, Li Y, Wu Y, Xu C, et al (2020) In vivo AAV-CRISPR/Cas9-mediated gene editing ameliorates atherosclerosis in familial hypercholesterolemia. Circulation 141, 67-79.

8. Sasaki Y, Ikeda Y, Miyauchi T, Uchikado Y, Uchikado Y, Ohishi M (2020) Estrogen-SIRT1 axis plays a pivotal role in protecting arteries against menopause-induced senescence and atherosclerosis. J Atheroscler Thromb 27, 47-59.

9. Wang J, He X, Chen W, Zhang N, Guo J, Liu J, Zhang L, Sun H, et al (2020) Tanshinone IIA protects mice against atherosclerotic injury by activating the TGF- $\beta /$ PI3K/Akt/eNOS pathway. Coron Artery Dis 31, 385-392.

10. Milgrom SA, Varghese B, Gladish GW, Choi AD, Dong W, Patel Z, Chung C, Rao A, et al (2019) Coronary artery dose-volume parameters predict risk of calcification after radiation therapy. $J$ Cardiovasc Imaging 27, 268-279.

11. Adam H, Eirini S, Konstantinos P, Akoumianakis E, Kosidekakis N, Kosidekakis N, Papadakis A, Hamilos M, et al (2020) CT-coronary angiography in asymptomatic male patients with high atherosclerosis risk: Is it justified? Hellenic J Cardiol 62, 129-134.

12. Vošmik M, Hodek M, Buka D, Sýkorová P, Grepl J, Paluska P, Paulíková S, Sirák I (2020) Cardiotoxicity of radiation therapy in esophageal cancer. Rep Pract Oncol Radiother 25, 318-322.

13. Xu Y, Li F, Zhao X, Tan C, Wang B, Chen Y, Cao J, Wu D, et al (2020) Methionine sulfoxide reductase A attenuates atherosclerosis via repairing dysfunctional HDL in scavenger receptor class B type I deficient mice. FASEB $J$ 34, 3805-3819.

14. Chen J, Zhang X, Millican R, Creutzmann JE, Martin S, Jun HW (2020) High density lipoprotein mimicking nanoparticles for atherosclerosis. Nano Converg 7, ID 6.

15. Shao J, Han J, Zhu Y, Mao A, Mao A, Zhang K, Zhang X, Zhang Y, et al (2019) Curcumin induces endotheliumdependent relaxation by activating endothelial TRPV4 channels. $J$ Cardiovasc Transl Res 12, 600-607.

16. Liu F, Fang S, Liu X, Li J, Wang X, Cui J, Chen T, Li Z, et al (2020) Omentin-1 protects against high glucoseinduced endothelial dysfunction via the AMPK/PPAR $\delta$ signaling pathway. Biochem Pharmacol 174, ID 113830.

17. Button EB, Gilmour M, Cheema HK, Martin EM, Martin EM, Robert J, Wellington C (2019) Vasoprotective functions of high-density lipoproteins relevant to alzheimer's disease are partially conserved in apolipoprotein Bdepleted plasma. Int J Mol Sci 20, ID 462.
18. Yuan R, Sun Z, Cai J, Yang X, Zhang W, Wu C, Shen Y, Yin A, et al (2020) A novel anti-cancer therapeutic strategy to target autophagy accelerates radiationassociated atherosclerosis. Int J Radiat Oncol Biol Phys 109, 540-552.

19. Bang OY, Chung JW, Lee MJ, Seo WK, Kim GM, Ahn MJ (2020) Cancer-related stroke: an emerging subtype of ischemic stroke with unique pathomechanisms. J Stroke 22, 1-10.

20. Ferguson AL, Kok LF, Luong JK, Van Den Bergh M, BellAnderson KS, Fazakerley DJ, Byrne SN (2019) Exposure to solar ultraviolet radiation limits diet-induced weight gain, increases liver triglycerides and prevents the early signs of cardiovascular disease in mice. Nutr Metab Cardiovasc Dis 29, 633-638.

21. Baselet B, Belmans N, Coninx E, Lowe D, Janssen A, Tabury K, Raj K, et al (2017) Functional gene analysis reveals cell cycle changes and inflammation in endothelial cells irradiated with a single X-ray dose. Front Pharmacol 8, ID 213.

22. Ramadan R, Vromans E, Anang DC, Goetschalckx I, Hoorelbeke D, Decrock E, Baatout S, Leybaert L, et al (2020) Connexin43 hemichannel targeting with TAT-Gap19 alleviates radiation-induced endothelial cell damage. Front Pharmacol 11, ID 212.

23. Ossoli A, Pavanello C, Giorgio E, Calabresi L, Gomaraschi M (2019) Dysfunctional HDL as a therapeutic target for atherosclerosis prevention. Curr Med Chem 26, 1610-1630.

24. Liu H, Zheng Y, Zhang Y, Li J, Fernandes S, Zeng D, Li $\mathrm{X}$, Schnaar R, et al (2020) Immunosuppressive Siglec$\mathrm{E}$ ligands on mouse aorta are up-regulated by LPS via NF- $\kappa B$ pathway. Biomed Pharmacother 122,ID 109760.

25. Getz GS, Reardon CA (2020) Atherosclerosis: cell biology and lipoproteins. Curr Opin Lipidol 31, 286-290.

26. Sutter I, Velagapudi S, Othman A, Riwanto M, Manz J, Rohrer L, Rentsch K, Hornemann T, et al (2015) Plasmalogens of high-density lipoproteins (HDL) are associated with coronary artery disease and anti-apoptotic activity of HDL. Atherosclerosis 241, 539-546.

27. Ruiz M, Okada H, Dahlbäck B(2017) HDL-associated ApoM is anti-apoptotic by delivering sphingosine 1phosphate to S1P1 \& S1P3 receptors on vascular endothelium. Lipids Health Dis 16, ID 36.

28. Guo S, Wang T, Zhang S, Chen P, Cao Z, Lian W, Guo J, Kang Y (2020) Adipose-derived stem cell-conditioned medium protects fibroblasts at different senescent degrees from UVB irradiation damages. Mol Cell Biochem $463,67-78$.

29. Cui X, Ma Y, Wang H, Huang J, Li L, Tang J, Cheng B (2020) The Anti-photoaging effects of pre- and posttreatment of platelet-rich plasma on UVB-damaged HaCaT keratinocytes. Photochem Photobiol 97, 589-599.

30. Theofilatos D, Fotakis P, Valanti E, Sanoudou D, Zannis V, Kardassis D (2018) HDL-apoA-I induces the expression of angiopoietin like 4 (ANGPTL4) in endothelial cells via a PI3K/AKT/FOXO1 signaling pathway. Metabolism 87, $36-47$.

31. Zheng A, Dubuis G, Ferreira CSM, Pétremand J, Vanli G, Widmann C (2019) The PI3K/Akt pathway is not a main driver in HDL-mediated cell protection. Cell Signal 62, ID 109347. 